\title{
Prestación sanitaria de calzado ortopédico en un área hospitalaria: Estudio transversal
}

\author{
Provision of orthopaedic footwear to patients in a hospital area: transversal study \\ José Luis Ledesma Vargas y Pedro García Fortea
}

Equipo provincial de Inspección de servicios sanitarios de Málaga

Palabras clave:

Dispositivos ortopédicos, zapatos, efectividad, calidad de vida, indicadores del estado de salud.
Keywords:

Orthotic devices, shoes, effectiveness, quality of life, health status indicators.
Recibido: 06-06-2019

Aceptado: 22-08-2019

\section{Resumen}

Introducción: Se aborda, con carácter preliminar, una brecha importante en la literatura con respecto a la efectividad del calzado ortopédico incluido como prestación sanitaria en España. Su objetivo fue describir las características de la prestación de calzado ortopédico a los pacientes en un área hospitalaria, y evaluar la validez de tres cuestionarios que miden la satisfacción del paciente (EQ-5D, SF-12 y FFI) para valorar la efectividad del calzado ortopédico, en particular comparando el calzado prefabricado con el calzado a medida.

Pacientes y métodos: Se evaluó las características de la prestación y la satisfacción del paciente con el calzado en muestra representativa de pacientes a los que se dispensó calzado ortopédico en un área hospitalaria de la provincia de Málaga ( $\mathrm{n}=29$ ).

Resultados: No se observaron diferencias significativas en las puntuaciones en los cuestionarios entre el calzado a medida y estándar, aunque en el grupo de calzado a medida fueron peores las puntuaciones mediante la escala FFI de la valoración funcional del pie, y de las dimensiones físicas y de dolor de los cuestionarios EQ-5D y SF-12. No obstante, la ausencia de diferencias se habría visto sesgada por la calidad del producto dispensado (el $42 \%$ de los pacientes a los que se prescribió calzado a medida habrían recibido calzado estándar), si bien se observaron valores significativamente menores mediante la escala visual analógica del EQ-5D en los pacientes con indicios de haber sido dispensado calzado estándar en vez del calzado a medida prescrito.

Conclusiones: Destacó la baja calidad del producto dispensado, recibiendo el 42 \% de los pacientes calzado estándar cuando les fue prescrito a medida. Aunque ello limita la validez de las puntuaciones obtenidas mediante los cuestionarios empleados, la escala visual analógica del EQ-5D permitiría discriminar los pacientes con indicios de dispensación de calzado estándar en vez del calzado a medida prescrito.

\section{Abstract}

Introduction: This study addresses, on a preliminary basis, an important gap in the literature regarding the effectiveness of orthopaedic footwear included as a health service in Spain. Its objective was to describe the characteristics of the provision of orthopaedic footwear to patients in a hospital area, and to evaluate the validity of three questionnaires that measure patient satisfaction (EQ-5D, SF-12, and FFI) to assess the effectiveness of orthopaedic footwear, in particular, comparing prefabricated footwear with custom footwear.

Patients and methods: Service characteristics and patient satisfaction with the footwear were evaluated in a representative sample of patients who received orthopaedic footwear in a hospital area of the province of Malaga $(n=29)$.

Results: No significant differences were observed in the scores on the questionnaires between the custom and standard footwear, although in the footwear group scores were worse for the functional assessment of the foot by FFI scale, and for EQ-5D and SF-12 physical and pain dimensions. However, the absence of differences would have been biased by the quality of the product dispensed ( $42 \%$ of the patients who were prescribed custom footwear would have received standard footwear), although significantly lower values were observed for the EQ-5D Analogue Visual Scale in patients who received standard footwear instead of the custom footwear prescribed.

Conclusions: The low quality of the product dispensed was relevant, receiving standard footwear the $42 \%$ of the patients who were prescribed custom footwear. Although this limits the validity of the scores obtained through the questionnaires used, the EQ-5D Analog Visual Scale would allow discriminating patients who received standard footwear instead of the custom footwear prescribed. 


\section{INTRODUCCIÓN}

En España los ciudadanos tienen derecho a un catálogo de prestaciones sanitarias que se hacen efectivas mediante un conjunto de técnicas, tecnologías o procedimientos (que se denomina cartera común de servicios del Sistema Nacional de Salud) basado en el conocimiento y experimentación científi$\mathrm{ca}^{1}$. Estas prestaciones incluyen la ortoprotésica, correspondiendo a un Comité Asesor formular las propuestas de actualización de esta cartera, y al médico de atención especializada la indicación y oportuna renovación de productos sanitarios para mejorar la calidad de vida y autonomía del paciente. La prestación ortoprotésica incluye los calzados ortopédicos dentro de las ortesis, al modificar las condiciones estructurales o funcionales del sistema neuromuscular o del esqueleto y estar adaptadas individualmente al paciente. Los calzados ortopédicos incluidos comprenden el calzado de plastazote o similar (en lo sucesivo, lo denominamos como calzado estándar) y el calzado a medida².

El calzado ortopédico a medida está catalogado como producto sanitario de la clase I, (productos no invasivos), es decir, se pueden realizar bajo la exclusiva responsabilidad del fabricante dado su bajo grado de vulnerabilidad y, por ello, no están sometidos a la declaración de conformidad de un organismo notificado. El calzado ortopédico a medida es el que se adapta a la forma y funciones del pie, y se realiza de acuerdo con una horma creada expresamente para un tipo de paciente. Suelen requerirlo los pacientes que tienen unas dimensiones antropométricas que están fuera de la normalidad o presentan grandes deformidades en sus pies, por lo que es imposible adaptarles un calzado ortopédico de serie. Su mecanismo de acción se basa en realinear la fuerza de reacción del suelo, redistribuyendo el peso mediante los principios biomecánicos de realineación del pie, en posición funcional, mediante un complejo sistema de fuerzas y su descarga, logrando reducir las presiones o fuerzas excesivas, mediante la realineación de la superficie plantar y el principio del contacto total entre la ortesis y el pie. Está indicado en grandes deformidades del pie que impiden el uso del zapato normal, pies zambos estructurados, equinos graves y otras deformidades que impidan la adaptación del calzado estándar. También en dismetrías importantes de miembros inferiores, en caso de trastornos neuropáticos o vasculares, etc. ${ }^{3}$. Para su elaboración es necesario el registro individual de mediciones. Aunque en la actualidad existen modernos sistemas mediante escáner en 3D (sistema CAD/CAM), la realidad es que este sistema es excepcional en las ortopedias, optándose por los métodos tradicionales de mediciones básicas, como el largo de la palmilla interior, altura de los dedos, perímetro metatarsal, del empeine, maléolos, altura de caña (bota), perímetro de caña a esa altura. Con posterioridad, se obtiene la horma mediante molde de escayola.

El calzado estándar (de plastazote en la norma legal) es un producto sanitario adaptado, que puede modificarse mediante pistola de calor (por estar compuesto por material termoconformable), consiguiendo adaptar el interior a las deformidades del pie (dedos en garra, hallux valgus, etc.). Su mecanismo de acción se basa en reducir las presiones en áreas dolorosas y acomodar el pie, constituyendo un lecho de descanso suave y de tacto agradable. Su uso es habitual en los pacientes con pie diabético, artríticos, neuropáticos y postquirúrgicos ${ }^{3}$.

Cada comunidad autónoma elabora el correspondiente catálogo de la prestación ortoprotésica y define las condiciones de acceso, de prescripción, de aplicación del citado catálogo y de gestión en su ámbito. Como ejemplo, en Andalucía se factura por calzado ortopédico más de 2.5 millones de euros al año por los calzados dispensados a más de 16 mil pacientes, atribuyendo a la Inspección de Servicios Sanitarios de la Junta de Andalucía la realización de programas de control de la prestación Ortoprotésica ${ }^{4}$, a través de sus equipos provinciales.

Nuestro equipo detectó casos de baja satisfacción entre los pacientes que emplearon calzado ortopédico correctamente indicado y dispensado. Para la medida de la satisfacción de los pacientes se dispone de herramientas adaptadas para población española, tanto de carácter genérico para calidad de vida (como el EuroQuol-SD y el Medical Outcomes Study 12-Item Short-Form Health Survey, abreviados en lo sucesivo como EQ-5D y SF-12, respectivamente), como específicas para valorar el dolor, discapacidad y limitación de la actividad de los pies (como el Foot Function Index, abreviado en lo sucesivo como FFI). No obstante, no se ha descrito en población española la efectividad ni la satisfacción de los pacientes que emplearon calzado ortopédico. Dada la trascendencia social y económica de esta prestación, nuestro equipo se propuso estimar la proporción de pacientes con baja satisfacción tras emplear calzado adecuado conforme a la indicación de los especialistas del Sistema Sanitario Público Andaluz, describiendo tanto las características de los pacientes como los factores que podrían determinar su satisfacción al tratamiento con calzado. Ello permitiría proponer criterios de indicación de calzado a medida que mejoren la eficiencia de esta prestación para los pacientes.

Este estudio está diseñado para abordar una brecha importante en la literatura de investigación con respecto a la efectividad del calzado ortopédico incluido como prestación sanitaria en España. Específicamente, el objetivo principal de este estudio es describir las características de la prestación de calzado ortopédico en un área hospitalaria, y evaluar la validez de los tres cuestionarios descritos (EQ-5D, SF-12, y FFI) para medir la efectividad del calzado ortopédico, en particular comparando el calzado prefabricado con el calzado a medida.

\section{PACIENTES Y MÉTODOS}

El presente estudio respondió a un diseño de carácter transversal exploratorio en un ámbito comunitario. Una vez 
se obtuvo la autorización del Comité de ética en investigación provincial de Málaga, se realizó el reclutamiento de participantes a partir del registro de facturas por calzado ortopédico en el Sistema Sanitario Público de Andalucía durante un año (entre 1 de agosto de 2016 y 31 de julio de 2017), facilitado por el Servicio de Conciertos del Servicio Andaluz de Salud. Fueron elegibles los pacientes de una de las seis áreas hospitalarias existentes en la provincia de Málaga (escogida al observar el menor número de calzados facturados en relación con su población asignada). Mediante un muestreo aleatorio simple, se seleccionó el $10 \%$ del total de 499 pacientes a los que se dispensó calzado ortopédico, que fueron citados para su reconocimiento durante el mes de noviembre de 2017. Los procedimientos utilizados en los pacientes fueron realizados tras obtener su consentimiento informado.

Como variables del estudio, se valoró tanto la indicación del calzado ortopédico, las características de la prestación (si se trataba de renovación, calidad del producto dispensado, y revisión por el facultativo prescriptor), así como su satisfacción. Los datos correspondientes a la indicación del calzado ortopédico se obtuvieron a partir de la información obrante en las historias clínicas del centro. Los pacientes fueron reconocidos en el hospital para analizar las características de la prestación y recoger información acerca de su satisfacción con el producto. La satisfacción se evaluó preguntándoles si habían notado alguna mejoría desde que utilizaban el calzado ortopédico, y mediante cuestionarios previamente validados en nuestra población para la calidad de vida en relación con la salud (EQ-5D 5 y SF-12 ${ }^{6}$ y la valoración funcional del pie (escala $\mathrm{FFl}^{7}$ ). Se resumen sus características principales de estos cuestionarios:

- El EQ-5D o EuroQol es un instrumento genérico de medición de la calidad de vida en relación con la salud, que puede utilizarse tanto en individuos relativamente sanos (población general) como en grupos de pacientes con diferentes patologías. El propio individuo valora su estado de salud, primero conforme a tres niveles de gravedad (sin problemas, algunos problemas o problemas moderados y problemas graves) en cinco dimensiones de salud (movilidad, cuidado personal, actividades cotidianas, dolor/ malestar, y ansiedad/depresión), y luego en una escala visual analógica de evaluación más general (de 0 a 10). Un tercer elemento del EQ-5D es el índice de valores sociales que se obtiene para cada estado de salud generado por el instrumento, no empleado en nuestro estudio.

- EI SF-12 consta de 12 ítems provenientes de las 8 dimensiones del SF-36 (dos de función física, uno de función social, dos de rol físico, dos de rol emocional, dos de salud mental, uno de vitalidad, uno de dolor corporal, y uno de salud general). Las opciones de respuesta forman escalas de tipo Likert que evalúan intensidad o frecuencia, oscilando el número de opciones de respuesta entre tres y seis dependiendo del ítem. En el análisis se emplearon las puntuaciones sumario físico y mental. Para facilitar la interpretación, estas puntuaciones se estandarizan con los valores de las normas poblacionales, de forma que 50 (desviación estándar de 10) es la media de la población general. Los valores superiores o inferiores a 50 deben interpretarse como mejores o peores, respectivamente, que la población de referencia. Para cada una de las 8 dimensiones, los ítems son codificados, agregados y transformados en una escala que tiene un recorrido desde 0 (el peor estado de salud para esa dimensión) hasta 100 (el mejor estado de salud), para ello se utilizó hoja de cálculo con los valores asignados.

- El FFI es un cuestionario que mide la escala de dolor, discapacidad y limitación de la actividad de los pies durante la semana anterior a la realización del mismo. La puntuación total consiste en dividir la suma de todos los ítems entre la calificación máxima obtenible: 207 (23 ítems × 9 puntos), y siendo después multiplicada por 100 para conseguir un porcentaje. Como resultado final, valores de porcentajes más bajos corresponden a un mejor índice de función del pie. Por el contrario, un FFI con valores cercanos al $100 \%$ se relaciona con una peor funcionalidad.

Como fuentes potenciales de sesgo se consideraron la edad, el sexo, la talla y peso de los pacientes.

Para analizar la indicación de calzado ortopédico se consideraron dos categorías: dolor o deformidad en las articulaciones del pie y otra patología. Se evaluó la calidad del producto dispensado al paciente detectando indicios de calzado estándar en vez de la realización de un producto sanitario a medida, al mostrar la marca del producto o el número del calzado, por las características de los componentes que configuran la construcción del calzado, así como por no disponer la ortopedia dispensadora los documentos de trazabilidad del calzado (proceso normalizado de trabajo y formularios empleados para la fabricación en la ortopedia, o, cuando se concertó con otro fabricante, la orden de taller, ficha técnica o factura del fabricante en la que constara el nombre comercial, modelo y sus componentes). Se analizó la distribución observada de estos grupos en función del tipo de calzado ortopédico, realizando las correspondientes tablas de contingencia y test de independencia mediante el test Chi cuadrado de Pearson.

Se confirmó que las variables cuantitativas seguían una distribución normal, comparando entonces las medias entre grupos (2 a 2) mediante el test t de Student. En caso contrario, se compararon sus rangos mediante el test no paramétrico $U$ de Mann-Whitney. Los datos fueron analizados con el programa Stata versión 15.1 (StataCorp, Texas, EE.UU.).

\section{RESULTADOS}

La Figura 1 muestra el proceso seguido para la selección de la muestra. La muestra de pacientes seleccionada estaba integrada por 37 mujeres con una edad media de 67 años, y 14 hombres con 61 años de edad media. Del total de 51 pacientes de la muestra, acudieron 29 (57\%). No se observaron diferencias significativas en relación con la edad y el sexo entre los pacientes que no acudieron y los reconocidos 


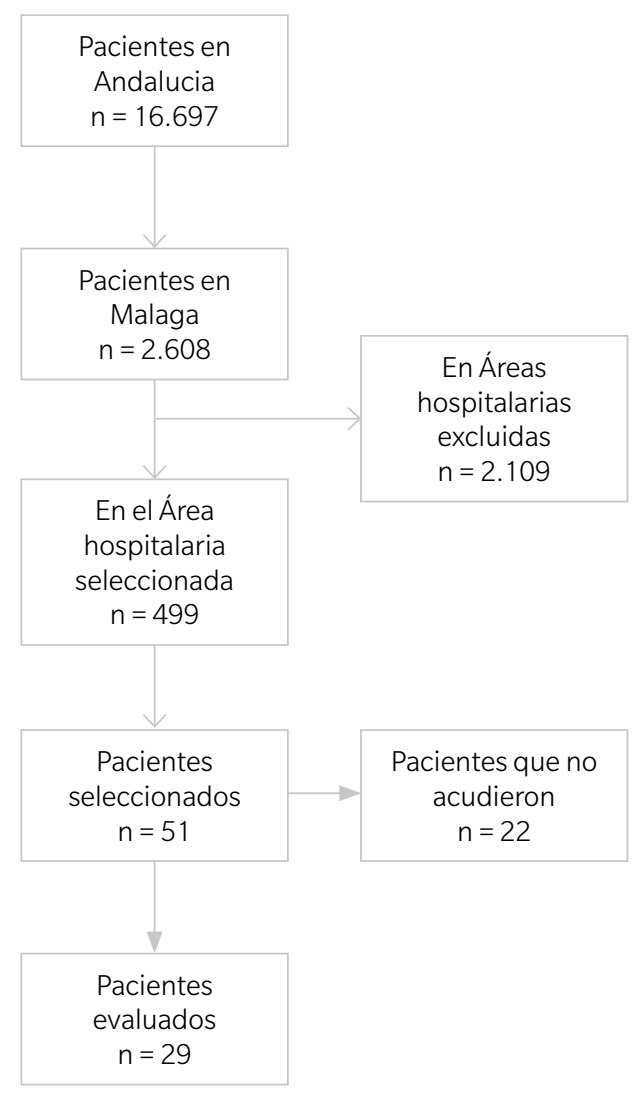

Figura 1. Diagrama de flujo seguido para la selección de la muestra.

en el hospital. No obstante, las mujeres acudieron proporcionalmente más que los hombres ( $62 \%$ de mujeres frente al $43 \%$ de hombres de la muestra), especialmente aquellas de mayor edad (69 años de edad media entre las mujeres que acudieron, mientras era de 64 entre las que no lo hicieron). En cambio, entre los hombres, apenas existían diferencias en la edad media de los que acudieron (60 años) y los que no (61 años).

Todos los casos evaluados (salvo uno que recibió calzado estándar o similar) se correspondieron con renovación de la prescripción. En todos los casos, el calzado ortopédico se acompañó además de plantilla. Generalmente $(n=28)$, la prescripción del calzado fue realizada por un traumatólogo, realizando el visto bueno del producto dispensado sin realizar su comprobación.

En la Tabla I se muestran las características individuales de los participantes en el estudio, y se comparan las mismas en función del tipo de calzado dispensado (a medida vs. estándar). Se observaron diferencias significativas entre los pacientes con calzado estándar y calzado a medida en relación con su peso ( $p=0.007 ; t=2.943,27$ g.l. $)$ y número de calzado $(p=0.017 ; t=2.551,27$ g.l.), con valores mayores en los pacientes con calzado a medida.
Por otro lado, se observaron diferencias significativas entre las dos categorías consideradas de indicación de calzado ortopédico (con dolor o deformidad en las articulaciones del pie y otra patología) en relación con el sexo $(p=0.016$; $\left.c^{2} \exp =5.7840\right)$ y talla del paciente $(p=0.035 ; t=-2.219$, 27 g.I.). Lo anterior se corresponde con las diferencias observadas entre hombres y mujeres en la talla $(p=0.026 ; t=2.363$, 27 g.I.), así como en el número de calzado $(p<0.005$; $\mathrm{t}=6.150,27 \mathrm{~g} . \mathrm{l}$.).

En relación con la satisfacción del paciente, al ser preguntados si habían notado alguna mejoría desde que utilizaban el calzado ortopédico, el $66 \%$ ( $n=8$ de 12$)$ de los usuarios de calzado estándar manifestó sentirse claramente mejor con el producto, un $17 \%$ manifiesta sentirse algo mejor, y un $17 \%$ no haber mejorado. El $94 \%(n=15$ de 16) de los usuarios de calzado a medida declaró sentirse claramente mejor con el producto, y un $6 \%$ manifiesta sentirse algo mejor $(n=1)$. No obstante, tales diferencias no resultaron estadísticamente significativas, como tampoco se observaron entre las categorías consideradas de indicación de calzado (Tabla I).

Considerando los cuestionarios validados para valorar la satisfacción de los pacientes (para la calidad de vida en relación con la salud mediante EQ-5D y SF-12, así como la valoración funcional del pie mediante la escala FFI), no se observaron diferencias significativas entre hombres y mujeres. No se advirtieron diferencias en las puntuaciones observadas entre las dos categorías consideradas de indicación de calzado ortopédico (con dolor o deformidad en las articulaciones del pie y otra patología) ni, en particular, entre los pacientes con calzado estándary calzado a medida (se muestra en la Tabla I).

Por último, en relación con la calidad del producto dispensado a los pacientes, en el $42 \%$ de los pacientes a los que se prescribió calzado a medida (5 de 12) se observaron indicios de calzado estándar en vez de la realización de un producto sanitario a medida. Mediante los instrumentos empleados para valorar la satisfacción entre ambos grupos de pacientes (con y sin indicios de calzado estándar), solo se observaron diferencias significativas en relación con la puntuación observada con la escala visual analógica del EQ-5D ( $p=0.020$; $\mathrm{t}=2.772,10 \mathrm{~g} . \mathrm{I}$.), con valores menores en los pacientes con indicios de calzado estándar.

\section{DISCUSIÓN}

Nuestros resultados son los primeros publicados en España sobre el impacto en la salud y calidad de vida de la población que recibe calzado ortopédico. Se ha descrito ${ }^{8}$ que el calzado a medida podría ayudar a mejorar la función, pero no el dolor en pacientes con diversas deformidades articulares de pie (en particular, pie cavo, artritis, fascitis plantar y hallux valgus doloroso).

En el área hospitalaria analizada, la prescripción de calzado fue realizada por médicos especialistas en Traumatología. Los pacientes a los que se prescribió calzado a medida eran de 
Tabla I. Características individuales de los participantes en el estudio.

\begin{tabular}{|c|c|c|c|c|}
\hline & $\begin{array}{c}\text { Pacientes evaluados } \\
(n=29) . \% \text { o media (DT) }\end{array}$ & $\begin{array}{c}\text { Calzado a medida } \\
(n=12) . \% \text { o media (DT) }\end{array}$ & $\begin{array}{c}\text { Calzado estándar } \\
(n=17) . \% \text { o media (DT) }\end{array}$ & Valor de $\mathrm{p}^{*}$ \\
\hline Edad (años) & $67.1(13.4)$ & $65.6(13.6)$ & $68.2(13.6)$ & n.s. \\
\hline Sexo & & & & n.s. \\
\hline Mujeres & $79.3 \%$ & $66.7 \%$ & $88.2 \%$ & \\
\hline Hombres & $20.7 \%$ & $33.3 \%$ & $11.8 \%$ & \\
\hline Peso (kg) & $66.7(12.2)$ & $73.7(11.6)$ & $61.7(10.2)$ & 0.007 \\
\hline Talla $(\mathrm{cm})$ & $162.9(9.5)$ & $165.1(8.9)$ & $161.4(9.9)$ & n.s. \\
\hline Patología por la que se indica & & & & n.s \\
\hline Deformidad articular del pie & $72.4 \%$ & $58.3 \%$ & $82.4 \%$ & \\
\hline Otra patología & $27.6 \%$ & $41.7 \%$ & $17.6 \%$ & \\
\hline Número de calzado & $38.6(2.1)$ & $39.6(2.2)$ & $37.8(1.7)$ & 0.017 \\
\hline Refiere alguna mejoría con calzado & & & & n.s. \\
\hline Sí & $82.1 \%$ & $75.0 \%$ & $87.5 \%$ & \\
\hline No & $17.9 \%$ & $25.0 \%$ & $12.5 \%$ & \\
\hline \multicolumn{5}{|l|}{ EQ-5 } \\
\hline Movilidad & & & & n.s. \\
\hline Sin problemas & $10.3 \%$ & $0.0 \%$ & $17.6 \%$ & \\
\hline Algunos o moderados problemas & $82.8 \%$ & $91.7 \%$ & $76.5 \%$ & \\
\hline Muchos problemas & $6.9 \%$ & $8.3 \%$ & $5.9 \%$ & \\
\hline Cuidado personal & & & & n.s. \\
\hline Sin problemas & $69.0 \%$ & $75.0 \%$ & $64.7 \%$ & \\
\hline Algunos o moderados problemas & $27.6 \%$ & $25.0 \%$ & $29.4 \%$ & \\
\hline Muchos problemas & $3.4 \%$ & $0.0 \%$ & $5.9 \%$ & \\
\hline Actividades cotidianas & & & & n.s. \\
\hline Sin problemas & $24.1 \%$ & $16.7 \%$ & $29.4 \%$ & \\
\hline Algunos o moderados problemas & $65.5 \%$ & $83.3 \%$ & $52.9 \%$ & \\
\hline Muchos problemas & $10.3 \%$ & $0.0 \%$ & $17.6 \%$ & \\
\hline Dolor/Malestar & & & & n.s. \\
\hline Sin problemas & $10.3 \%$ & $0.0 \%$ & $17.6 \%$ & \\
\hline Algunos o moderados problemas & $58.6 \%$ & $66.7 \%$ & $52.9 \%$ & \\
\hline Muchos problemas & $31.0 \%$ & $33.3 \%$ & $29.4 \%$ & \\
\hline Ansiedad/Depresión & & & & n.s. \\
\hline Sin problemas & $41.4 \%$ & $41.7 \%$ & $41.2 \%$ & \\
\hline Algunos o moderados problemas & $37.9 \%$ & $50.0 \%$ & $29.4 \%$ & \\
\hline Muchos problemas & $20.7 \%$ & $8.3 \%$ & $29.4 \%$ & \\
\hline Estado de salud & & & & n.s. \\
\hline Sin problemas & $17.2 \%$ & $8.3 \%$ & $23.5 \%$ & \\
\hline Algunos o moderados problemas & $20.7 \%$ & $8.3 \%$ & $29.4 \%$ & \\
\hline Muchos problemas & $62.1 \%$ & $83.3 \%$ & $47.1 \%$ & \\
\hline Escala visual analógica (0-10) & $5.0(1.8)$ & $5.3(1.1)$ & $4.9(2.2)$ & \\
\hline \multicolumn{5}{|l|}{ SF12 } \\
\hline Sumario de puntuación física & $36.5(7.4)$ & $34.1(5.9)$ & $38.2(8.1)$ & n.s. \\
\hline Sumario de puntuación mental & $38.0(8.7)$ & $41.1(9.0)$ & $35.8(8.0)$ & n.s. \\
\hline FFI & $51.8(24.6)$ & $59.5(16.1)$ & $46.4(28.3)$ & n.s. \\
\hline Días de dolor & $4.8(2.5)$ & $5.8(1.8)$ & $4.1(2.7)$ & n.s. \\
\hline
\end{tabular}

DT: desviación típica. * Valor de $p$ al comparar las características individuales de los pacientes con calzado a medida y calzado estándar 
mayor talla que los de calzado estándar, observando ser de menor edad, con mayor proporción de hombres y de patología diferente a la deformidad articular del pie. En relación con la calidad del producto dispensado a los pacientes, el $42 \%$ de los pacientes a los que se prescribió calzado a medida habrían recibido calzado estándar.

En relación con los cuestionarios empleados (EQ-5D, SF-12, y FFI) para valorar la efectividad del calzado prescrito, no se observaron diferencias significativas entre el calzado a medida y estándar, si bien en el grupo de calzado a medida se observaron peores puntuaciones de la valoración funcional del pie mediante la escala FFI y para las dimensiones físicas y de dolor de los test de calidad de vida en relación con la salud. No obstante, la ausencia de diferencias se habría visto sesgada por la calidad del producto dispensado. De hecho, en los pacientes con indicios de haber sido dispensado calzado estándar en vez del calzado a medida prescrito, se observaron valores significativamente menores mediante la escala visual analógica del EQ-5D. La calidad del calzado a medida dispensado estaría condicionada al realizar su fabricación un proveedor diferente al establecimiento de ortopedia que lo dispensa. Para ello, la ortopedia envía los registros individuales del paciente al proveedor, pudiendo realizar bien su fabricación completa (así como posteriormente las correcciones necesarias), bien su fabricación parcial remitiendo los componentes del calzado a la ortopedia donde son ensamblados.

Nuestro estudio tiene limitaciones que afectarían a la validez de los resultados observados en relación con la efectividad del producto sanitario prescrito. Además de la deficiente calidad descrita del producto dispensado, casi todos los casos fueron renovaciones de la prescripción, por lo que desconocemos el estado funcional de partida con el que comparar el resultado del tratamiento con calzado ortopédico. Por otro lado, dado el carácter preliminar del estudio, el escaso tamaño de la muestra de pacientes y su composición heterogénea en relación con la patología que determinó la indicación del calzado ortopédico, dificultó observar diferencias significativas entre grupos de pacientes. Otros autores ${ }^{9}$ intentan evitar estas limitaciones empleando criterios restrictivos de selección de los pacientes en función de su patología (artritis reumatoide) y mediante una asignación aleatoria de los pacientes para recibir calzado a medida o estándar, sin poder disponer aún de resultados concluyentes.

Creemos que se debería profundizar en el estudio de la efectividad clínica y coste del calzado estandarizado y per- sonalizado en personas cuya patología lo requiere, así como el momento y diseños que pueden generar mejores resultados terapéuticos. Ello ayudará a elaborar un modelo de prescripción, dispensación, y posterior evaluación del resultado observado con este tipo de ortesis.

\section{CONFLICTO DE INTERESES}

Los autores declaran no tener ningún conflicto de intereses.

\section{FINANCIACIÓN}

El presente estudio no dispuso de financiación, formando parte de un Acuerdo de colaboración en la Evaluación de Prestaciones Sanitarias previsto en el Plan anual de Inspección de servicios sanitarios de 2018 (aprobado por Orden de la Consejera de Salud de 13 de febrero de 2018), realizado por el Equipo provincial de Málaga.

\section{BIBLIOGRAFÍA}

1. Ley $16 / 2003$, de 28 de mayo, de cohesión y calidad del Sistema Nacional de Salud.

2. Real Decreto $1030 / 2006$, de 15 de septiembre, por el que se establece la cartera de servicios comunes del Sistema Nacional de Salud y el procedimiento para su actualización.

3. VV. AA. Guía descriptiva de Ortoprótesis: Ortesis de miembro superior y miembro inferior. Tomo II. Madrid: Ministerio de Sanidad y Consumo - Centro de publicaciones; 2001. Disponible en: https://www.mscbs. gob.es/profesionales/prestacionesSanitarias/CarteraDeServicios/ ContenidoCS/6PrestacionOrtoprotesica/docs/GuiaDescriptivaOrtoprotesisTomo2.pdf.

4. Orden de 13 de febrero de 2018, por la que se aprueba el Plan Anual de Inspección de Servicios Sanitarios. Boletín Oficial de la Junta de Andalucía 2018 (Número 38:22).

5. Badia X, Roset M, Montserrat S, Herdman M, Segura A. [The Spanish version of EuroQol: a description and its applications. European Quality of Life scale]. Med Clin (Barc). 1999;112 Suppl 1:79-85.

6. Alonso J, Regidor E, Barrio G, Prieto L, Rodriguez C, de la Fuente L. [Population reference values of the Spanish version of the Health Questionnaire SF-36]. Med Clin (Barc). 1998;111(11):410-6.

7. Paez-Moguer J, Budiman-MakE, Cuesta-Vargas Al. Cross-cultural adaptation and validation of the Foot Function Index to Spanish. Foot Ankle Surg. 2014;20(1):34-9. DOI: 10.1016/j.fas.2013.09.005

8. Hawke F, Burns J, Radford JA, du Toit V. Custom-made foot orthoses for the treatment of foot pain. Cochrane Database Syst Rev. 2008(3):CD006801. DOI: 10.1002/14651858.CD006801.pub2.

9. Gallagher KS, Godwin J, Hendry G], Steultjens M, Woodburn J. A protocolfor a randomised controlled trial of prefabricated versus customised foot orthoses for people with rheumatoid arthritis: the FOCOS RA trial [Foot Orthoses - Customised v Off-the-Shelf in Rheumatoid Arthritis]. J Foot Ankle Res. 2018;11:24. DOI: 10.1186/s13047-018-0272-3. 